\title{
DISTROFIAS DEL PRIMER CUATRIMESTRE
}

\author{
Drs. ADRIANA JORQUERA, ARTURO GALLO y ADALBERTO STEEGER
}

\author{
Cátedra de Pediattía de! Prof. Adalberto Steeger. \\ Hospital "San Juan de Dios". Santiago.
}

Distrofia es una alteración crónica de la asimilación celular que tiene lugar en el período del lactante, período que nosotros convencionalmente extendemos hasta los 2 años y, en ciertos casos, más allá.

Según la terminología de la escuela alemana, la distrofia corresponde a los trastornos nutritivos crónicos. Diferenciamos estos trastornos crónicos de los trastornos nutritivos agudos que se caracterizan fundamemialmente por alteraciones en el metabolismo hidrosalino. En cambio los crónicos se caracterizan por las alteraciones provocadas por el deficiente aprovechamiento de las substancias sólidas: proteínas, hidratos de carbono, grasas $y$ vitaminas.

Czerny y Finkelstein recalcan la etiología alimenticia de estos 2 grupos de trastornos, colocando las infecciones y la constitución en 2 ? Iugar. Ellos observaron que los distróficos, aún aquellos muy semejantes en su morfología, tienen reacciones muy diversas frente a los alimentos e infecciones. Este diversos comportamiento del distrófico, sin poder siempre señalar una lesión anatómica objetiva, Finkelstein lo describió como la "reacción funcional del lactante", reacción que pasa a ser el elemento directivo en el tratamiento y pronóstico de estos trastornos nutritivos crónicos. En esta reacción intervienen, además de la calidad del alimento, la infección y la constitución del lactante.

En nuestro medio proletario, Ios defectos en las dietas son muy notorios, agregándose el ambiente antihigiénico en que estos lactantes viven $\mathrm{y}$ se desarroIllan.

La constitución influye no sólo a través de las desviaciones de la normalidad, como: malformaciones congénitas, diátesis, etc., sino que lo hace fundamentalmente a través de la edad, puesto que el lactante, en los 2 primeros años, va cambiando en su reacción frenie al medio ambiente, representado por la alimentación e infección.
Por este tan destacado rol que desempeña la edad, Scroggie y Wiederhold han propuesto, muy acertadamente, el encasillamiento de las distrofias en diversos grupos de acuerdo con la edad, a saber: niños de 1 a 4 meses; de 4 a 8 meses; de 8 a 14 y de 14 a 24 meses y más.

En un sentido estricto es difícil hablar de distrofias en el primer mes de la vida. Las distrofias en esie período son raras $\mathrm{y}$ pueden atribuirse a malformaciones congénitas, diátesis y sepsis del recién nacido. Ya en los meses siguientes, las distrofias se empiezan a ver con más frecuencia, presentando en el primer cuatrimestre algunas características, que han sido resumidas por varios autores en los siguientes pumios:

I. Existe la hipoalimentación simple que no ha dañado la capacidad de alimentación celular o lo ha hecho débilmente, y que debe diferenciarse de la distrofia. Es útil observar la reacción a la alimentación adecuada a su edad y peso, con la cual el hipoalimentado simple muestra una rápida recuperacóin, lo que no sucede con el distrófico. Como se comprende, la distinción es sólo retrospectiva. Los antecedentes de presencia o ausencia de factores constriucionales e infecciosos, también contribuyen a la diferenciación.

II. El potencial de crecimiento se mantiene, salvo en casos de causa hereditaria o congénita, de parte del niño o de notoria carencia materna, durante la gestación, o alimentación muy deficiente en próieínas en la vida extrauterina.

III. Las avitaminosis no se manifestarían clínicamente en este período.

IV. La capacidad inmunitaria es escasa o nula por falta de maduración del sistema reticulo-endotelial. I LOS elementos defensivos específicos son de origen materno trasmitidos a iravés de la placenta. Por tanto, ciertas infecciones tienden a la generalización.

V. El mayor porcentaje de agua del organismo infantil, que en su mayor proporción es extracelular, favorece la acen- 
tuada trofo, hidro y termolabilidad de las distrofias de este período; y en segundo lugar permite que infecciones, aún relativamen'e localizadas, tengan repercusión metabólica general mayor que en cualquier otra edad.

Sin embargo, la revisión de la literatura revela una muy escasa preocupáción por el estudio de las características de la distrofia en las distintas edades del lactante en general, y del primer cuatrimestre de la vida en especial.

Como parte de un estudio más amplio sobre la imporiancia del proceso de crecimiento y desarrollo en los distintos procesos fisiológicos y patológicos del lactante, hemos abordado el estudio de las dis. trofias en el lactante menor.

\section{MATERIAL Y MÉTODO DE THABAJO}

Nuestro trabajo consistió en la revisión de las fichas clínicas de 100 lactantes ingresados con menos de 4 meses de etad y con el diagnóstico de distrofia, al Servicio de Pediairía del Hospital "San Juan de Dios", en los años $195 \bar{a}$ a 1957. Las 100 fichas utilizadas fueron entregadas por la Oficina de Estadística, sin selección alguna. Los datos contenidos en ella fueron vaciados a un formulario común, con espacio para la siguiente información:

1. Identificación: nombre y ficha.

2. Antecedentes familiares y ambientales: edad, salud y ocupación de los padres; previsión, No de orden y sitio de atención del parto, tipo de vivienda, $N$ ? de personas por pieza y cama.

3. Antecedentes personales: peso de nacimiento, con'trol médico e inmunizaciones, alimentación y progreso ponderal anteriores a la hospitalización, enfermedades anteriores.

4. Ex. físico de ingreso: edad, peso, talla y circunferencias, déficit ponderal y estatural, signos físicos de distrofia y diagnóstícos concomitantes.

5. Exámenes de laboratorio practicados en el hospital. talarios.

6. Alimentación y tratamientos hospi-

7. Evolución: progreso del peso y talla, enfermedades intercurrentes, tiempo de normalización de los signos físicos $\mathrm{y}$ exámenes de laboratorio, tiempo de estada.
8. Egreso: edad, peso, talla, causa de ahia, indicaciones dadas, autopsia en caso de fallectmiento.

9. Evolución posterior al alta y comentario general.

A partir de este formulario, se confeccionaron tablas para los diferentes datos analizados. Además, se confeccionó una tarjeta perforada para cada uno de los 100 lactantes en estudio, que se utilizaron para establecer tablas de correlación entre los dis intos rubros consignados en el formulario. F1 total de tablas fabricadas en esta forma alcanzó a 178, cuvo contenido es la base de la comunicación presente.

\section{RESULTADOS}

Los presentaremos clasificados en los rubros de antecedentes, examen de ingreso, tratamiento $y$ evolución.

1. Antecedentes: Ei mayor número de lactantes tuvo un peso normal de nacimierro, con un $16 \%$ de prematuros. Es posible que esta proporción de prematurez haya sido mayor, ya que en 23 de los 100 niños no se consignaba este dato en la ficha. Fué curioso observar que en los prematuros la distrofia avanzada fué menos frecuente que en los niños de término. En cambio, el pronóstico vital fué en ellos frecuentemente malo, a pesar de un control post-alta superior a los otros lactantes.

El esiudio del número de onden del parto demostró que el $65 \%$ de los casos son terceros hijos o inferiores y el $45 \%$ ocupan el $5^{\circ}$ lugar o superiores, lo que es más alto que en la pobilación en general. Se observó además que los hijos de familias numerosas nacieron en menor proporción en maternidades, tuvieron peor control médico, presentaron distrofias más avanzadas y su evolución fué menos favorakile.

EI antecedente deI sitio de atención deI parto no mostró diferencias importantes con la población general, ya que el $62 \%$ de los partos ocurrió en maternidad y sólo el $9 \%$ no tuvo atención profesional. EI parto en maternidad fué índice de un mayor sentido de responsabilidad de la familia, coincidiendo con una mejor sunervisión médica, anterior $\mathrm{y}$ posterior a la hospitalización, pero no demostró re- 
lación con la gravedad ni evolución de la distrofia.

Ev un $30 \%$ de las fichas no existía informacin sobre control médico anterior a la hospitlaización, y en el $\mathbf{5 9} \%$ habia algún tipo de atención. Los niños sin a'iención médica recibieron en general alimentación artificial más tardíamente que los controlados. Es curioso que este retraso en la complementación alimenticia parece haber sido desfavorable, ya que el ingreso de estos niños fué en promedio más precoz y con un déficit ponderal más acentuado que en los lactantes con supervisión médica. El análisis de los dátos no permite deducciones sobre diferencias en gravedad y pronóstcio del cuadro según control médico.

La importancia de la alimentación al pecho resalta en el hecho que sólo un $10 \%$ de los lactantes seguúa con alimentación materna exclusiva en el momento de ingreso, el $72 \%$ inició la alimentación artificial antes de cumplir los 2 meses y el $59 \%$ en el 1er. mes. Observamos que el grupo de niños con alimentación exclusiva al pecho ingresaron más precozmente, presentaron una dis'rofia más avanzada en general y su pronóstico fué peor.

Los antecedentes mórbidos se presentaron en la mayoría de los casos, ya que sólo el $29 \%$ no los tuvo. Los más frecuentes fueron diarreas infantiles $(23 \%)$, respiratorias $(11 \%)$, patología del recién nacido ( $7 \%$ ) y otros. En el $15 \%$ de los casos había antecedentes de enfermedades repetidas, sobre todo de trasiornos nutritivos agudos. La existencia de patología anterior no mostró relación directa con la gravedad y evolución de la distrofia.

Respecto a los antecedentes familiares y amtientales, ellos figuraban sólo en. la menor proporción de las fichas, por lo que no hemos podido evaluar su importancia. La edad de los padres fué favorable en la mayoría, ya que el $82 \%$ de los padres y el $84 \%$ de las madres tenían enire 20 y 40 años. Sóio un $7 \%$ de los padres eran empleados o comerciantes y el resto obreros, cesantes o sin información. La salud de los padres fué satisfactoria en general.

La suficiencia de la alimentación anterior a la hospitalizacion no pudo ser determinada, por la vaguedad y falta de ve- racidad de las informaciones de los familiares al respecto.

2. Clínica: La distribución de la edad de ingreso fué la siguiente:

1er. mes

$2^{\circ}$ mes $6 \%$

3er. mes $19 \%$

4 ? mes

Sin datos $42 \%$ $32 \%$

TOTAL $100 \%$

No hubo diferencias importantes en las características clínicas de la distrofia de los lactantes ingresados a diferentes edades, salvo una menor frecuencia de anemia en los menores de 1 mes. En cambio, la evolución fué desfavorable en este grupo de niños, mejorando al aumentar la edad de ingreso.

El peso y talla de ingresos se presentan en la Tabla 1:

TABIA NO 1

PESO Y TAILA DE INGRESO DE 100 LACTAN'IES MENORES DE 4 MESES CON DIAGNOSTICO DE
DISTROFIA

Rospltal San Juan de Dlos, 1854-1957

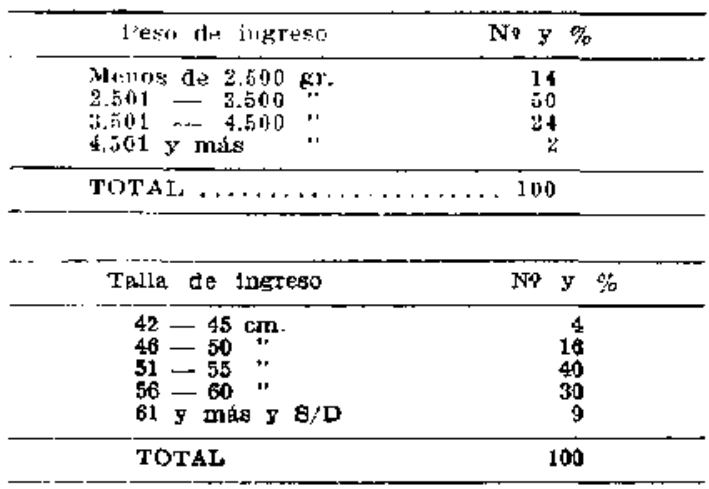

De acuerdo con el criterio de la Cátedra, se clasificaron las distrofias en grados I, II y III, según que el déficit del prooeso ponderal fuera de $10-33 \%, 34-$ $66 \%$ o $67 \%$ y más al ingreso. El $50 \%$ de los casos correspondió a distrofia III, el $28 \%$ a II, el $8 \%$ a I y sólo un $6 \%$ no pudo ser clasificado. El grado de distrofia demostró ser un criterio útil de clasificación, ya que los grados avanzados preserviaron mayor sintomatología y mayor compromiso de la talla. Sin embargo, no se observó relación con el pronóstico. 
Ya hemos visto que Ias distrofias más avanzadas se presentaron con mayor frecuencia en los lactantes sin control médi$c o$, en aquellos que mantenían alimentacin materna exclusiva y los que presen. taban antecedentes de patología del recién nacido.

El déficit de talla se calculó sobre la base de un aumento ideal de $3 \mathrm{~cm}$. mensuales. En el $26 \%$ de Ios casos, la talla fué normal, el aumento tuvo un déficit de $10-33 \%$ en el $17 \%$, de $34-66 \%$ en el $20 \%$ y de $67 \%$ o más en el $15 \%$. Uno de los hechos más categóricos de nuestra observación fué que los lactantes ingresados con talla normal, presentaron una evolución francamente más favorable que la de los niños con déficit estatural. El progreso ponderal fué más rápido, presentaron menos enfermedades intercurrentes y los períodos de peso estacionario y de hospríalización fueron más cortos en aquéllos que en éstos. El pronóstico fué también mejor, con un $8 \%$ de mortalidad intra y post hospitalaria controlada, contra un $27 \%$ en los que tenían talla muy disminuída. La disminución de talla presentó también una relación directa clara con el número de orden del parto y el déficit ponderal.

Las circunferencias corporales estuvieron, en general, bastante comprometidas. El $36 \%$ tenia circunferencia craneana de $35 \mathrm{~cm}$. o menos y el $58 \%$ entre $31 \mathrm{y}$ $38 \mathrm{~cm}$. El $51 \%$ presentaba circunferencia toráxica de 32 ó $33 \mathrm{~cm}$. El déficit mayor, empero, se presentó en la circunferencia abdominal, que midió $31 \mathrm{~cm}$. menos en el $51 \%, 32-35 \mathrm{~cm}$ en el $26 \%$ y 36 o más cm. sólo en el $11 \%$.

Los signos físicos de distrofia los clasificamos en signos corrientes, signos avanzados y otros. Los signos corrientes incluyen la disminución del panículo adiposo, palidez, turgor disminuído, hiper o hipotonía muscular. Los 2 primeros fue. ron los más frecuentes, preserríndose en más de la mitad de los casos (56\% y $55 \%$ ), la hipertonía muscular $(19 \%)$ fué bastante más frecuente que la hipotonía (9\%). Signos avanzados se consideraron la existencia de edemas y signos cutáneomucosos de carencias. Los edemas se observaron en el $11 \%$ de los niños y los signos carenciales en el $20 \%$. De los otros signos, el $16 \%$ presentó compromiso acen- tuado del estado general y decaimiento, el $13 \%$ hepatomegalia, el $16 \%$ signos óseos radiológicos (osteoporosis, retraso crecimiento, raquilismo, escorbuto) y el $6 \%$ trastornos circulatorios. Sólo 7 lactantes no presentaron otro signo que el déficit ponderal.

En general, la existencia de signos avanzados y decaimiento fué índice de peor evolución y de pronóstico. Los lactantes que sólo presentaron los signos corrientes ingresaron, en una mayor proporción, por otros cuadros patológicos agregados. Ya hemos visto que los signos avanzados de distrofia se vieron con mayor frecuencia en los hijos de familias numerosas y en los sin control médico anterior, y con menor frecuencia en aquellos que habían iniciado precozmente una alimentación artificial o que tenían talla normal.

Todos los lactantes del grupo analizado ingresaron con otra afección, siendo la más corriente los trastornos nutritivos agudos $(68 \%)$, respiratorios $(27 \%)$, infecciosos (22\%), prematurez $(16 \%)$, malformaciones congénítas $(16 \%)$ y cutáneas (22\%). De estas últimas, llamó la atención la frecuencia de la dermatitis seborroide $(11 \%)$, afección que apareció además frecuentemente como enfermedad intercurrente en el curso de la hospitalización. Otras afecciones presentes fueron raquitismo, trastornos neurológicos, anemia y propias del recién nacido. Esta patología corresponde bastante bien con la distribución general de los diagnósticos de ingreso en un Servicio de Lactantes. Llamó la atención que Ios lactantes ingresados con dermatitis seborroide hicieron, en general, una peor evolución.

De los exámenes de laboratorio sólo el hemograma se practicó en un número suficientemente alto de niños como para permizir conclusiones. El 30\% de los lactantes presentó una anemia al ingreso, entendiendo por tal una cifra inferior a 3.500 .000 rojos y sólo un $32 \%$ tuvo más de 4.000.000. Las anemias se presentaron con mayor frecuencia en los niños con dermatitis seborroide y su presencia significó evolución más arrastrada, pero no un peor pronóstico final. Un alto porcentaje de hemogramas mostró anisocitosis $(74 \%)$, macrocitosis $(43 \%)$ y poiquilocitosis $(18 \%)$, en tanto que la microcito- 
sis, hipocromia, policromatofilia y megalocitosis fueron excepcionales. Sólo el $7 \%$ tenían los glóbulos rojos de caracteres normales.

La serie blanca dió una leucocitosis en el $51 \%$ de los casos, con desviación a la izquierda sólo en el $28 \%$. La RTDN fué muy frecuente.

3. Tratamiento. EI tratamiento dietético se hizo, en general, según el esquema de la Cátedra, con algunas modificaciones individuales. Se inició con Eledón al $10 \%$,en cantidades de $50-100 \mathrm{cc} / \mathrm{kg} /$ día, según la gravedad del cuadro. Se aumentó luego la cantidad hasta $150 \mathrm{cc}$. por $\mathrm{kg}$. de peso ideal, con un máximo de 180 $200 \mathrm{cc}$. por $\mathrm{kg}$. de peso real. Finalmente seaumentó la concentración con hidratos de carbono hasia un 10 a $12 \%$, o se agregó Czerny hospitalario, pasando luego a la alimentación normal para la edad. Casi la totalidad de los niños recibió además vitamina $C$, la mitad complejo $B$, un $42 \%$ golpe vitamínico $\mathrm{D}$ y un $20 \%$ vitaminas $\mathrm{A}$ y $\mathrm{D}$ en gotas diarias.

Sólo el $8 \%$ no recibió antibióticos 0 sulfas. Los más usados fueron, en este mismo orden, el cloranfenicol, penicilina, estreptomicina, sulfas y tetraciclinas. En el $33 \%$ de los casos se combinaron 2 o más de ellos. En la mitad de los casos, el tiempo total de suministro de antibióticos fué inferior a 10 días, en el $22 \%$ de 11 a 20 y en el $22 \%$ de 21 y más días. Hubo una relación inversa evidente entre la prolongación del tiempo de antibióticos y la evolución de la distrofia, ya que los lactantes que recibieron antibióticos por tiempo prolongado progresaron mal de peso, tuvieron mayor número de enfermedades intercurrentes y prolongaron su estada considerablemenie. Esta observación no autoriza a obtener ninguna conclusión sobre relación de causa a efecto, ya que es obvio que los tratamientos más prolongados corresponden a los casos más graves.

Se colocó flebo o gastroclisis al ingreso a 36 lactantes, repitiéndose este tratamiento en 11 de ellos en el curso de la hospitalización. 74 niños recibieron transfusiones de sangre, la mayoría en forma de una serie de 3 transfusiones día por medio y 20 con series repetidas. 26 recibieron transfusiones de plasma. Los lactantes que recibieron transfusiones eran en general los más graves, por lo que el proceso ponderal de ellos fué más lento, al igual que el periodo de peso estacionario.

4. Evolución y pronóstico: La evolución se midió por el progreso ponderal y estatural, enfermedades intercurrentes, días de estada y pronóstico final.

El progreso ponderal se inició en el $34 \%$ de los casos junto con el ingreso, en ianto que en el $17 \%$ de los casos se mantuvo estacionario de 1 - 10 días y en el $37 \%$ más de 11 días, prolongándose en 7 casos más de 30 dias y en 10 por toda la hospitalización. La prolongación del período de peso estacionario significó siempre un mal pronóstico, una evolución arrastrada, hospitalización larga y letalidad alta.

El aumento de peso fué lento en general, con un promedio menor de $10 \mathrm{gr}$. por día de hospitalización en el $52 \%$ de los casos y de más de $30 \mathrm{gr}$. sólo en el $16 \%$. El buen aumento ponderal se acompañó siempre de un pronóstico francamente más favorable, en tanto que el progreso deficiencie correspondió a la letalidad $y$ morbilidad hospitalaria altas $y$ hospitalizaciones prolongadas. Repetimos que los lactantes ingresados en el primer mes de vida tuvieron un progreso más lento y peor pronóstico. El déficit estatural mostró también relación con la rapidez del progreso ponderal, pero no así el déficit ponderal de ingreso. La existencia de anemia y de signos de distrotia avanzada coincidieron también con un progreso deficiente. Según la forma de progreso del peso, clasificamos muestros casos en 5 tipos de curvas, que son las siguientes:

Tipo 1, estacionaria, $10 \%$ de los casos; Tipo 2, ascendente inmediata, $40 \%$ de los casos; Tipo 3 , descendente, $14 \%$ de los casos; Tipo 4, ascendente tardía, $20 \%$ de Ios casos; Tipo 5, ascendente detenida, $5 \%$ de los casos.

La aparición de enfermedades intercurrentes en el curso de la hospitalización se presentó en el $68 \%$ de los casos, siendo las más frecuentes las respiratorias (en 36 lactantes), nutritivas agudas (30), cutáneas (46), piurias (2) y otras (55). 16 niños presentaron 2 enfermedades intercurrentes y 30 presentaron 3 o más, hasta 6 ó 7. La aparición de estas enfermedades a repetición estuvo ligada a una peor evolución general $y$ a una mayor letalidad. 
El período de hospitalización fué de menos de 14 días en el $35 \%$ de los casos, de 15-30 en el $24 \%$ y sobre 30 en el $41 \%$, llegando en 9 casos a más de 60 días. Ya hemos visto la relación de estos hechos con los otros índices de evolución y sólo cabe hacer resaltar la importancia administrativa de estas cifras.

De los 100 lactantes estudiados, 70 salieron de alta por mejoría de la patología aguda agregada, 6 por razones administra'ivas y 14 por fallecimiento. De ellos, 41 no recibieron indicaciones de alta y al resto se le instruyó sobre alimentación y necesidad de control externo. EI $49 \%$ del total de lactantes no volvieron ni se tiene información de ellos, lo que impide sacar conclusiones sobre la evolución posterior al alta. De los 32 que volvieron a control, 4 más fallecieron hasta el momento del trabajo. Hubo 15 que hicieron una evolución posterior satisfactoria con recuperación parcial o total de su distrofia, y 13 una evolución desfavorable, con persistencia de la distrofia, morbilidad a repetición y reingreso al hospital en varios de ellos. Repetimos que la letalidad hospitalaria fué más alta en los niños con mayor déficit de calla, con anemia al ingreso, con menor progreso ponderal y con mayor número de enfermedades intercurrentes. También se apreció relación con el bajo peso de nacimiento, falta de control médico anterior, introducción tardía de la alimentación artificial y antecedentes de patología deI período del recién nacido.

5. Evaluación de las distrofias: En nuestra Cátedra es cos'umbre evaluar las distrofias según 4 criterios: grado o diagnóstico morfológico, antiguiedad, etiología y reacción funcional. Ya hemos informado sobre el diagnóstico morfológico de nuestros distróficos. E1 diagnóstico cronológico fué muy difícil de determinar con presición, pudiendo establecer solamente que el $25 \%$ eran recientes y el $63 \%$ aníguas, de las cuales el $17 \%$ desde el nacimiento.

La reacción funcional, que se aprecia por la respuesta a la alimentación y terapéutica adecuadas, fué catalogada como mala en el $31 \%$ de los casos, regular en el $25 \%$ y buena en el $40 \%$. Reconocemos que el criterio usado para esta clasifica- ción fué eminentemente subjetivo $\mathrm{y}$ está sujeto a discusión.

Finalmente, respecto al diagnóstico etiológico, se comprobó la existencia del factor carencial en la mayoría de los niños $(70 \%)$, del factor infecioso en el $54 \%$, constitucional o congénito en el $37 \%$ y otros dudosos en el $38 \%$. La mayor parte de las veces, los distintos factores aparecieron combinados, por lo que resultó difícil delimitar su imporiancia real.

\section{DISCUSIóN}

Existe consenso unánime sobre la escasa frecuencia de la aparición de distrofias en el 1.er mes de la vida. Es interesante, por eso, que en nuestro material, el $6 \%$ ocurrieron en esta edad.

En ellos habían seguramente factores constitucionales importantes y estos ninos fueron, en general, los de peor pronóstico. La prematurez fué el factor que se demośró como de mayor importancia.

Según la clasificación morfológica, e] $86 \%$ de Ios casos fueron distrofias de grado II y III, pero este hecho no guardó relación con el pronóstico de la distrofia. En esto estamos de acuerdo con Scroggie y Wiederhold, quienes piensan de que en el primer cuatrimestre las distrofias, aún graves, no se traducen en modificaciones morfológicas más o menos proporcionadas.

Inversamente, la talla demostró ser un índice de pronóstico mucho más seguro. Comprobamos además, que el déficit de aumento de talla fué evidente en la mayor parie de los niños, sobrepasando el $67 \%$ de déficit en el $15 \%$ de los casos, lo aue contradice la opinión clásica que la talla no se afecta en las distrofias de los lactantes menores.

Contrariando también las opiniones de otros autores, nosotros encontramos signos de carencia vitamínica cutánea y mucosa en el $20 \%$ de los casos, y aún en un $11 \%$ hubo aparición de edemas. Es posible que todos estos hechos estén señalando una carencia alimenticia intra $y$ extrauterina muy acentuada.

Tiene valor destacar la alta incidencia de dermatitis seborroide como antecedente de la hospitalización o como afección intercurrente, lo que tal vez pueda ser un argumento a favor de la teoría que 
explica la dermatitis seborroide como una carencia en biotina.

Los difererries índices usados para medir la evolucóin y pronóstico de la distrofia coincidieron en la mayor parte de los casos (progreso pondo-estatural, enfermedades intercurrentes, dias de estada), de modo que podria usarse cualquiera de ellos, pero el que parece más práctico es el progreso ponderal.

Un aporte de interés de nuestra observación, fué que nos permitió clasificar el progreso ponderal en 5 tipos de curvas, que a nuestro entender abarcan todas las posibilidades.

La disergia presente en es'os niños se confirmó por la alta incidencia de enfermedades intercurrentes.

La cifra de mortalidad fué más o menos igual a la de la población hospitalaria general de lactantes, pero este hecho está seguramente alterado porque no conocemos con exactitud el pronóstico vital post-hosprialario.

En los pocos casos en que se pudo seguir el control post-hospitalario, el pronóstico fué malo en general, demostrando el peso del factor ambiental en la producción y mantención de estos cuadros.

En general, los resultados de nuestro análisis nos han indicado que la distro. fia en el primer cuatrimestre de la vida, no presenta diferencias fundamentales con la de las otras edades, excepto en los aspectos que recién hemos considerado.

\section{RESUMEN}

1. Se hacen algunas consideraciones sobre las características del lactante menor y su posible influencia con las distrofias de esta edad.

2. Se revisan las fichas de 100 lactantes ingresados al Servicio de Pediatría del Hospital "San Juan de Dios" entre 1955 - 1957, con menos de 4 meses de edad y con diagnóstico de distrofia.

3. La revisión efectuada permitió destacar los siguierries hechos de interés:

a) Antecedentes: La prematuridad fué un aporte etiológico importante. Las distrofias más graves y de peor pronóstico se presentaron en hijos de familias numerosas, con supervisión médica deficiente y en que la alimentación complementaria fué más diferida. La hipoalimentación por leche materna fué un fenómeno generalizado.

b) Clínica: Contrariamente a to esiablecido por otros autores, el progreso estatural deficiente fué un fenómeno frecuente, y demostró una relación mucho más estrecha con el pronóstico que el déficit ponderal. Los otros signos físicos de mayor frecuencia fueron la pérdida del panículo adiposo, la palidez y la disminución del turgor de la piel. Los signos cutáneo-mucosos de carencias vitamínicas y los edemas se presentaron en un número importanie de casos. Todos los lactantes ingresaron con otro cuadro patológico. La dermatitis seborroide se presentó con una frecuencia apreciable.

c) Evolución: Los diferentes índices de evolución usados coincidieron en la mayoría de los casos, siendo el más práctico el aumento de peso. Se clasificaron todas las formas de progreso ponderal observadas en 5 tipos de curva de peso. La mortalidad intrahospital fué de $14 \%$, semejante a la mortalidad general de los lactantes hospi:alizados.

El control post-alta de la evolución no se pudo efectuar en buena forma, pero los casos analizados mostraron un pronóstico alejado deficiente.

4. Se discuten algunos aspectos de los hechos observados y se concluye que las diferencias de las distrofias del lactante menor con las otras edades, son menores que las semejanzas.

\section{SUMMARY}

DYSTROPHIES IN THE FIRST FOUR MONTHS OF LIFE.

1) Some considerations are made on the characteristics of the young infant and their possible influence on the dystrophies of this age.

2) The records of 100 infants hospitalized in the pediatric ward of the San Juan de Dios Hospital between 1955 and 1957, aged less than 4 months and diagnosed as dystrophic ones, are reviewed.

3) The performed survey permited to detach the following interesting facts:

A) Antecedents: Prematurity was an important etiological contribution. The most serious cases and with bad prognosis appeared in children of numerous families with deficient medical watch 
over and whose complementary feeding had been most postponed. The inadequale breast-feeding was a general phenomenon.

B) Clinic: In contrast to the findings of other authors, the deficient progress of growth in length was a frequent phenomenon and showed a much closer relation to the prognosis than the deficit of weight. The other physical signs of greatest frequency were: loss of the pannicuIus adiposus, pallor and diminution of the skin turgor. The cutaneous and mucosal signs of vitamin deficiencies and oedema manifested themselves in an important number of the cases. All the infants entered to the hospital with another pathological picture. Seborrheic dermatitis had among them an appreciable frequency.

C) Course: The different indices used for the judgment of the course coincide in the majority of the cases, the increase of weight being the most practical one. All the forms of weight progress observed were classified into 5 types of weight curves. The mortality rate within the hospital amounted to $14 \%$, similar to that of the general mortality rate of the hospitalized infants.

The follow-up of the course could not be carried out satisfactorily, but the analyzed cases showed a bad late prognosis.

$4)$ Some aspects of the observed fac:are discussed and it is concluded that tho diferences between the dystrophies of tha younger infants and those of the othe: ages are less than the similarities.

\section{ZUSAMMENFASSUNG}

DYSTROPHIEN IN DEN ERSTEN VIER LEBENSMONATEN.

1) Einige Betrachtungen über die $\mathrm{Be}$ sonderheiten des jüngeren Säuglings und ihren möglichen Einffluss auf die Dystrophien in dieser Altersstufe werden angestellt.

2) Die Krankenblätter von 100 weniger als 4 Monate alten Säuglingen, die mit der Diagnose Dystrophie in den Jahren 1955 bis 1957 auf die Abteilung für Kinderkrankheiten des San Juan de Dios Krankenhauses aufgenommen wurden, werden durchgesehen.

3) Die vorgenommene Durchsicht gestattete, folgende Tatsachen von Interesse hervorzuheben:
A) Vorgeschichte: Frühgeburt war ein erheblicher Beitrag zur Aetiologie. Die schwersten und prognostisch ungünstigsten Dystrophien traten bei Kindern aus vielköpfigen Familien auf, die ärztlich ungenügend überwacht waren, und bei denen der Uebergang zur Beikost am längsten hinausgeschoben war. Unzureichende Ernährung mit Muttermilch war eine allgemeine Erscheinung.

B) Klinik: Im Gegensatz zu den Fesistellungen anderer Autoren war das Zurüickbleiben im Längenwachstum eine häufige Erscheinung und zeigte eine viel engere Beziehung zur Prognose als das Gewichtsdeficit. Die häufigsten anderen körperlichen Krankheitszeichen waren: Verlust des Panniculus adiposus, Blässe und verminderter Turgor der Haut. Hautund Schleimhautzeichen von Vitaminmängeln, sowie Oedeme zeigten sich in einer erheblichen Zahl der Fälle. Alle Säuglinge boten bei der Aufnahme noch ein anderes Krankheitsbild. Eine seborrhoische Dermatitis bestand mit beträchtlicher Häufigkeit.

C) Verlauf: Die verschiedenen Indices für die Beurteilung des Verlaufs stimmen in der Mehrzahl der Fälle überein; unter ihnen, wat die Gewichtszunahme der praktischste. Alle beobachteten Formen der Gewichtsentwicklung wurden in 5 Typen von Gewichtskurven klassifiziert. Die Sterblichkeit innerhalb des Krankenhauses fbetrug $14 \%$, also etwa so viel wie die allgemeine Sterblichkeit der jm Krankenhaus behandelten Säuglinge.

Die Nachkontrolle des Verlaufs konnte nicht in zufriedenstellender Form durchgeführt werden, aber die Fälle, in denen eine Nachuntersuchung möglich war, zeigten auf längere Sicht eine ungünstige Prognose.

4) Dia beabachteten Tatsachen werden unter verschiedenen Gesichtspunkten diskutiert, und es wird gefolgert, dass die Unterschiede zwischen den Dystrophien der jüngeren Säuglinge und denen der anderen Altersstufen geringer sind als die Aehnlichkeiten.

\section{BIBLIOGRAFÍA}

1.-CIENFUEGOS, E. - "Trastornos Nutrltivos Croricos del Lactante". Rev. Chllema Pedlat. 10:629, 1939. 2.-CZEFANY, A. - Cit. por Clenfuegos, $\mathbf{E}$.

3.-FINKIELSTEIN, H. - "Tratado de las Enpermedades del lactante". Ea. Labor. Barcelona. 1941.

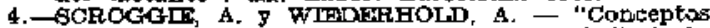
modernos en el tratamiento de la Distrofia". Amh. de Ped. del Uruguay. $26: 484$, 1965. 